\title{
Clinical Presentation of Pulmonary Tuberculosis in 10-19 Year (Adolescent) Patients
}

\author{
Dr Mani Shankar, Dr Partha Kumar Chaudhuri ${ }^{2}$, Dr Anil Kr Chaudhary ${ }^{3}$ \\ Junior Resident ${ }^{1}$, Associate Professor ${ }^{2}$, Professor ${ }^{3}$. \\ Department of Pediatrics, Rajendra Institute of Medical Sciences, Ranchi, Jharkhand, India
}

\begin{abstract}
Background:-Tuberculosis continues to be the one of the most important infectious cause of morbidity and mortality in India. Lungs are the portal of entry of M.Tuberculosis in the body. Lungs are the commonest site for tuberculosis disease. The disease in lungs varies from a small parenchymal lesion to disseminated disease.

Aims and Objectives:-The objective of the study is to analyse the clinical presentation of pulmonary tuberculosis in adolescent (10-19 years). As against younger patients in whom extra pulmonary tuberculosis $(T . B)$ is seen, adolescent usually have pulmonary lesions with an adult pattern of presentation.

Material and Methods:-It was a cross-sectional hospital based study with random selection study group. 138 adolescent patients visiting the OPD of RIMS, Ranchi hospital were included in the study. The gender of the patient, past history of tuberculosis, history of Koch's contact, sputum smear results, x-ray features and management were taken into consideration.

Results: - Out of 138 patients, 78 were girls and 60 were boys. All were between 10-19 years of age. Of all the 138 patients the $x$-ray features showed fibro-cavitory apical lesions in 58(42\%) cases. 44(32\%) were sputum positive and majority of them were girls. A significant number had history of Koch's contact.

Conclusion: - Adolescent females are more susceptible to pulmonary tuberculosis than males. Sputum smear positivity is found in a large proportion in adolescents. On radiology, adolescent have adult pattern of disease demonstrated by presence of apical cavitory lesions
\end{abstract}

Keywords: Adolescent, Pulmonary Tuberculosis, Chest X-Ray

\section{Introduction}

India is the highest TB burden country in the world in terms of absolute number of incident case that occurs each year. It accounts for one-fourth of the estimated global incident TB cases in 2015. ${ }^{1,2}$ Although childhood tuberculosis is rarely recorded with accuracy, children contribute substantially to the global TB disease burden. Many factors are associated with the risk of acquiring M.Tuberculosis infection.

There is striking age related risk of developing tuberculosis following primary infection and the age related difference in the disease manifestations. Childhood tuberculosis can be divided into intrathoracic and extrathoracic TB. Most children with TB develop pulmonary TB but recognition of extrathoracic TB is equally important.

Tuberculosis is often missed or overlooked due to non-specific symptoms and difficulties in diagnosis. Unlike in adults, diagnosis of tuberculosis in children is difficult due to paucibacillary nature of the disease and due to lack of characteristic complications like cavity lesions, fibrosis etc. So tuberculosis should never be diagnosed on the basis of chest $\mathrm{x}$-ray alone. In children diagnosis is based on history, H/O contact, positive tuberculin test, $\mathrm{x}$ ray features and if possible isolating the organism. Demonstration of mycobacterium in various clinical specimens remains gold standard, this is often not possible due to paucibacillary nature of the illness.

In children with presumptive Pediatric TB, every attempt must be made to microbiologically prove diagnosis through examination of appropriate respiratory /non-respiratory specimen with quality assured diagnostic tests. ${ }^{3}$

\section{Material And Methods}

This study was done in a tertiary centre teaching hospital in Jharkhand during period of Jan 2016 to Dec 2016. The subjects of study were selected from children (Age 10-19 years) visiting OPD of hospital with complain of cough and/or fever for more than 2 weeks were included in the study. Exclusion criteria was cough and/or fever less than 2 weeks, age less than 10 years and greater than 19 years and children already on ATT. Total 138 adolescent patients visiting OPD were included in the study. The gender of the patient, past history of tuberculosis, history of Koch's contact, sputum smear results, $\mathrm{x}$ ray features and management were taken into consideration. 


\section{Result}

Out of 138 patients $60(43.5 \%)$ were boys and $78(56.5 \%)$ were girls( Figure-1).All patients were between 10-19 years of age.Fibrocavitory lesion was found in $58(42 \%)$ patients.44 patients (32\%) were sputum positive.88 patients $(63.7 \%)$ had history of Koch's contact.90 patients $(65 \%)$ had history of prior antibiotic treatment. None of them had taken anti tuberculous treatment prior to our hospital visit.

In the age group (10-13 years) there were total 31 patients out of which 10(32.3\%) had cavitory lesion on chest x-ray, 04(12.9\%) had positive sputum smear.14(45.2\%) patients in this age group had history of Koch's contact.( Table-1) In age group 14-17 years there were total 45 patients out of which 18(40\%) had cavitations on chest x-ray, 10(22.2\%) had positive sputum smear.30 (66.7\%) had history of Koch's contact. In the age group $18-19$ years there were 62 patients. 30(48\%) had cavitations on chest x-ray and 30(48.4\%) had positive sputum smear. 44(70.9\%) patients had history of Koch's contact.

Figure-1: Showing gender distribution.

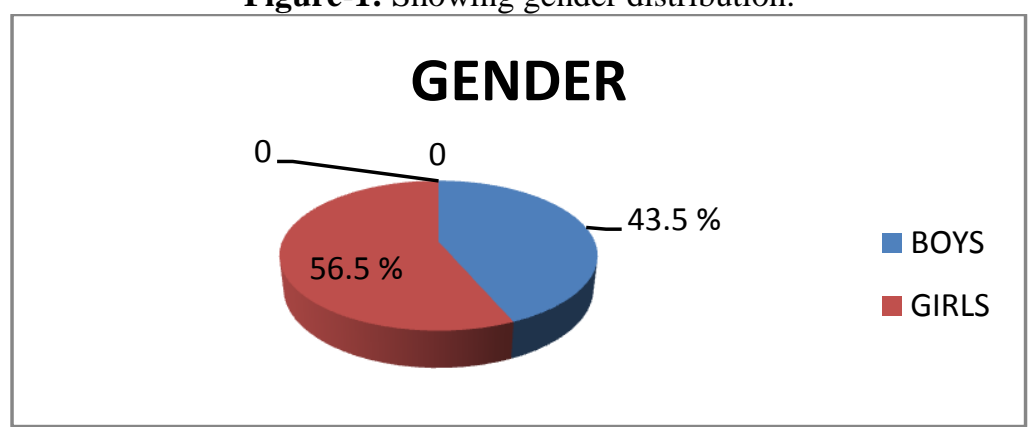

Table-1: Showing clinical presentation of pulmonary tuberculosis in 10-19 year (adolescent) patients

\begin{tabular}{|l|l|l|l|l|}
\hline AGE GROUP & 10-13 YEARS & 14-17 YEARS & 18-19 YEARS & TOTAL \\
\hline NO $(\%)$ & $31(22.5)$ & $45(32.6)$ & $62(44.9)$ & 138 \\
\hline CAVITATION ON CHEST X-RAY (\%) & $10(32.3)$ & $18(40)$ & $30(48.4)$ & $58(42)$ \\
\hline SPUTUM SMEAR POSITIVE (\%) & $04(12.9)$ & $10(22.2)$ & $30(48.4)$ & $44(32)$ \\
\hline KOCH'S CONTACT (\%) & $14(45.2)$ & $30(66.7)$ & $44(70.9)$ & $88(63.7)$ \\
\hline
\end{tabular}

Percentage in top row shows percentage of all children presenting at different ages

Percentage in other rows refers to percentage of children at each age presenting with a particular feature.

\section{Discussion}

The Immature immune response in very young children (2-3 years of age) are at high risk of developing progressive disease while children (3-10 years) are the least likely to progress to disease after primary infection. ${ }^{4}$ After this period there is sudden there is sudden increase in the risk of progressing to disease following primary infection during adolescence. This is what leads to so called adult type of disease i.e. development of adult type pulmonary cavitation. In this study most severe lesions were related to longer duration of symptoms possibly due to diagnosis delay in health services. Adolescent girls are more prone to tuberculosis probably due to poor nutrition; lesser seeking of health care and their involvement in case of other pulmonary TB cases at home makes them more vulnerable to acquire the disease. In our study majority [78(56.5\%)] of the patients were girls. Actual prevalence among girls could be even higher as Jharkhand being a state with low literacy less number of infected female child reach the health centre for seeking treatment. Another limitation of our study was the small sample size.

Marais et al. reported eight children (10-14 years) who developed adult type cavitating disease which could be diagnosed by sputum smear microscopy in contrast to younger children for whom smear microscopy has very little diagnostic value. ${ }^{5}$ Diagnostic workup of extrapulmonary TB in adolescent girls as a routine also must have an USG of abdomen to rule out TB of reproductive organs to avoid damage to genital organs which is a cause of significant long-term reproductive morbidity in women. ${ }^{6}$

This study had some limitations. One concern was the small number of sample size of the patient. Also due to poor literacy among the patients and social stigma related to tuberculosis it was difficult to elicit the contact history which might have affected the result. Also it was difficult to analyse the social condition of the adolescent based on data given by them.

\section{Conclusion}

To conclude most cases of TB in adolescents were similar to those in adults: apical pulmonary lesions with cavitations. Primary TB forms were rare. ${ }^{7}$ There is a need to diagnose these cases as early as possible especially among females to decrease morbidity and mortality.

$\begin{array}{lll}\text { DOI: } 10.9790 / 0853-1605094547 & \text { www.iosrjournals.org } & 46 \mid \text { Page }\end{array}$




\section{References}

[1] Govt. of India (2016), Revised National TB Control Programme Technical and operational Guidelines for Tuberculosis control in India,2016, Ministry of Health and Family Welfare, New Delhi.

[2] K. Park, Park's Textbook of preventive and social medicine. $24^{\text {th }}$ edition. BanarsidasBhanot Publishers, (2016) 206-8.

[3] Int. J. Tuberculosis Lung disease 2009, Dec 13 (12):1566-8

[4] Essentials of tuberculosis in children V.Seth, S.K.Kabra. $4^{\text {th }}$ edition, 105-107

[5] Marais BJ, Gie RP, HesselingAH,et al. Adult type Pulmonary Tuberculosis in Children 10-14 years of age. The Pediatric Infectious Disease Journal. 2005;24:743-4

[6] Kumar S. Female Genital Tuberculosis In: Sharma SK,Mohan A (Eds). Tuberculosis, $2^{\text {nd }}$ edn. Delhi: Jaypee Medical Publishers (Pvt) Ltd.,2009;463-78

[7] Sant'Anna CC, March MF, Barreto M, Pereira S, Schmidt C. Pulmonary tuberculosis in adolescents: radiographic features. Int J Tuberc Lung Dis. 2009;13:1-3 